\title{
The Small Scale Effect on the Dynamic Behavior of Cantilever and Fixed-Fixed Nano-Switches
}

\author{
B. Chen, L. Tan, and Z. Y. Ou
}

\begin{abstract}
Based on the conventional Euler bucking model and the effective nonlocal elasticity theory, the dynamic behaviors of cantilever and fixed-fixed nano-switches are studied in this paper. The governing equations for the dynamic response of the nano-switches including small-scale effect are derived by using the energy variational method. The small-scale effects on the stiffness of nano-switches are presented. It is found that the scale effect has softened the fixed-fixed nano-switches and hardened the cantilever nano-switches.
\end{abstract}

Index Terms-Scale effect, cantilever nano-switch, fixed-fixed nano-switch, effective nonlocal elasticity.

\section{INTRODUCTION}

With the development of nano-electro-mechanical system (NEMS), nano-switch as an important building block in NEMS is gaining interest among researches. Typically, a nano-switch contains fixed and deformable structures, separated by a dielectric medium. When a voltage is applied between the deformable and fixed structures, electrostatic forces induced charges and intermolecular produced the surface absorption act on both structures. Since the fixed structure cannot move, the movable structure tends to approach to the fixed one, and elastic force tends to take the movable structure back into the undeformed position. Exceeding the applied voltage above a certain value makes the movable electrode to collapse onto the fixed structure [1]. Nano-switch usually includes two cases such as cantilever and fixed-fixed.

When the dimensions of nano-switch are small and comparable to molecular distances, the size effects are significant in their mechanical behavior [2]. Therefore, size dependent theories of continuum mechanics have received increasing attention in recent years due to the need to model and analyze very small sized mechanical structures and devices [3]. Ordinarily, the classical continuum mechanics is not suitable for interpreting the size-dependent behaviors of nanostructures. Since controlled experiments in nano-scale are difficult and molecular dynamic simulations are computationally expensive [4], the non-classical continuum theories such as nonlocal elasticity [5] or couple-stress [6] had been set up and used to explain the size-dependent behaviors of nanostructures. Y. Tadi Beni, M. R. Abadyan,

Manuscript received May 26, 2014; revised August 6, 2014. This work was supported in part by the National Natural Science Foundation (Grant No. 11062004 and 11362009), the Fundamental Research Funds for Universities of Gansu Province and the Returned Overseas Scholars Fund in Gansu.

B. Chen and L. Tan are with the School of Mechanical and Electronical Engineering, Lanzhou University of Technology, Lanzhou 730050, China (e-mail: chenbo7126@126.com, 18394024767@163.com ).

Z. Y. Ou is with the School of Science, Lanzhou University of Technology, Lanzhou 730050, China (e-mail: zhiyingou@163.com).
Aminreza Noghrehabadi [7] applied the modified couple-stress theory incorporating with the modified Adomian decomposition solving method to investigate the size dependency of the pull-in characteristics of NEMS. Their results revealed that the size effect increases the pull-in voltage of the nano-beams. In order to account the small-scale effect, J. Yang, X. L. Jia and S. Kitipornchai [8] investigated the pull-in instability of nano-switches subjected to the electrostatic force produced by the applied voltage and intermolecular force (van der Waals and Casimir forces) within the framework of nonlocal elasticity theory. They proposed a linear distributed load (LDL) model to obtain closed form solutions for cantilever and fixed-fixed nano-switches. They found that in cantilever nano-switches, an increase in the small scale factor leads to a higher pull-in voltage, whereas in fixed-fixed nano-switches, it leads to a lower pull-in voltage. J. S. Peng, G. B. Luo, L. Yang and J. Yang [9] modified the LDL model using a linear load model which suits boundary conditions and restudied the pull-in instability of nano-actuators by using Eringen's nonlocal elasticity theory. Also, B. L. Wang, S. J. Zhou, J. F. Zhao and $X$. Chen [10] presented a size-dependent model for electrostatically actuated microbeam using strain gradient elasticity theory. T. Mousavi, S. Bornassi and H. Haddadpour [11] had dealt with the pull-in behaviors of nano-switches using Eringen's nonlocal elasticity theory. Through differential quadrature method, K. F. Wang and B. L. Wang [12] investigated the pull-in instability of a nano-switch under electrostatic and intermolecular Casimir forces.

In order to investigate the nonlocal small scale effect for details, the effective nonlocal elasticity theory was introduced which was based on energy variational method and the nonlocal elasticity theory. According to the nonlocal elasticity theory which was built by Eringen [13], not only the strain field at that point but also the strains of all the other points of the body determine the stress field at an arbitrary point in a continuum body. Concretely speaking, an arbitrary point in a continuum body will produce a strain filed which will affect other points of the continuum body, that is, the stress field at an arbitrary point is determined by all the strains in the continuum body.

The drift of this investigation is first to obtain the governing equation of the nano-switches subjected to electrostatic and intermolecular Casimir forces, and then gradually to solve the ordinary differential equation covered. The approach here, however, is analytic.

\section{THE EFFECTIVE NONLOCAL ELASTICITY MODEL FOR CANTILEVER AND FIXED-FIXED NANO-SWITCHES}

\section{A. The Model of Cantilever Nano-Switches}

Cantilever nano-switches, being a kind of classical nano- 
switches, consist of a movable plate and a fixed plate. Under a critical "pull-in" voltage, the cantilever becomes unstable or pulls-in onto the fixed plate. In order to study the dynamic behavior of the nanostructure, we should consider the Casimir force, the van der Waals force and the electrostatic force, together.

As what shown in the Fig. 1, it's the model of the cantilever nano-switch which we study in this paper.

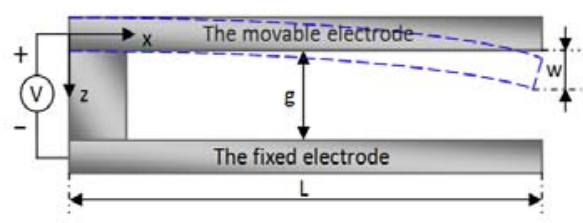

Fig. 1. The model of cantilever nano-switch.

\section{B. The Model of Fixed-Fixed Nano-Switches}

In the Fig. 2, it's the model of fixed-fixed nano-switch which we study in this paper.

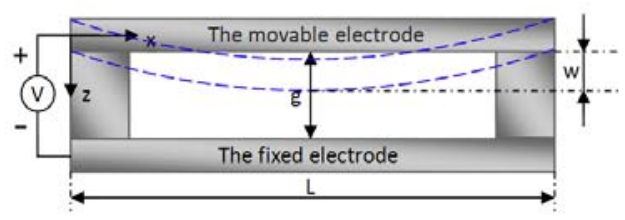

Fig. 2. The model of fixed-fixed nano-switch.

There is some parameters covered in the cantilever and fixed-fixed nano-switches model, $L$ is the length of nano-switch, $V$ is the applied voltage, $x, z$ are the axial coordinate and the transverse coordinate, respectively.

\section{The Effective Nonlocal Theory Model}

According to the Euler-Bernoulli beam theory, the relation- ship between the displacement along $x$ and the strain $\varepsilon_{x x}$ can be written as

$$
\varepsilon_{x x}=-z \frac{\partial^{2} w(x, t)}{\partial x^{2}}
$$

where $t$ is the time, $\varepsilon_{x x}$ is the normal strain and the origin of coordinate is at the leftmost of the moveable plates.

In order to simplify the calculation and embody the nonlocal nanoscale effects, the nonlocal nanoscale parameter $\tau$ is expressed as

$$
\tau=\frac{e_{0} a}{L}
$$

where $\mathrm{e}_{0} a$ is the parameter given by matching against experiments and molecular dynamic simulations.

Under two dimensional cases, the stress-strain relationship based on the Green's function for the nano-switches can be written as [5]

$$
\left(1-\tau^{2} L^{2} \nabla^{2}\right) \sigma_{i j}=\sigma_{i j}^{\prime}
$$

where $\sigma_{i j}$ is the nonlocal stress and $\sigma_{i j}^{\prime}$ is the classical stress and $\nabla$ is the Hamiltonian operator.

When it comes to the one dimensional stress-strain relationship for the nano-switches, the nonlocal stress-strain relationship is

$$
\sigma_{x x}-\tau^{2} L^{2} \frac{\mathrm{d}^{2} \sigma_{x x}}{\mathrm{~d} x^{2}}=E \varepsilon_{x x}
$$

where $\sigma_{x x}$ is the nonlocal stress for the axial coordinate, $E$ is the modulus of elasticity.

In order to facilitate theoretical formulations, it is convenient to nondimensionalize (4) with the following substitutions,

$$
\bar{\sigma}_{x x}=\frac{\sigma_{x x}}{E}, \tau=\frac{e_{0} a}{L}, \bar{x}=\frac{x}{L}, \bar{z}=\frac{z}{L}, \bar{w}=\frac{w}{L}
$$

The equation (4) changes to

$$
\bar{\sigma}_{x x}-\tau^{2} \frac{\partial^{2} \bar{\sigma}_{x x}}{\partial \bar{x}^{2}}=\varepsilon_{x x}
$$

or

$$
\bar{\sigma}_{x x}-\tau^{2} \frac{\partial^{2} \bar{\sigma}_{x x}}{\partial \bar{x}^{2}}=-z \frac{\partial^{2} \bar{w}}{\partial \bar{x}^{2}}
$$

The equation (4) or (5) is a two order ordinary differential equations, the general solution can be expressed as

$$
\bar{\sigma}_{x x}=B_{1}(\bar{z}) e^{\bar{x} / \tau}+B_{2}(\bar{z}) e^{-\bar{x} / \tau}+\sum_{n=1}^{\infty} A_{n} \frac{\partial^{2 n} \bar{w}}{\partial \bar{x}^{2 n}}
$$

where $B_{1}(\bar{z}), B_{2}(\bar{z})$ and $A_{n}$ are the integral constants.

It can be derived from (6) and (7) that the constant coefficient $A_{n}$ is

$$
A_{n}=-\tau^{2 n-2} \bar{z}
$$

Generally speaking, when nonlocal nanoscale parameter goes to zero $(\tau \rightarrow 0)$, the nonlocal stress $\sigma_{x x}$ gradually becomes the classical stress $\sigma_{x x}^{\prime}$, that means $\lim _{\tau \rightarrow 0} \bar{\sigma}_{x x}=\varepsilon_{x x}$. Thus, the integral constants of the general solution must be zero $\left(B_{1}(\bar{z})=B_{2}(\bar{z})=0\right)$. Then the solution of the nonlocal stress can be written as

$$
\bar{\sigma}_{x x}=-\sum_{n=1}^{\infty} \tau^{2 n-2} \bar{z} \frac{\mathrm{d}^{2 n} \bar{w}}{\mathrm{~d} \bar{x}^{2 n}}=\sum_{n=1}^{\infty} \tau^{2 n-2} \frac{\mathrm{d}^{2 n-2} \varepsilon_{x x}}{\mathrm{~d} \bar{x}^{2 n-2}}
$$

The strain energy density $\mu$ of the per unit volume after deformation is

$$
\mu=\int_{0}^{\varepsilon_{x x}} \sigma_{x x} d \varepsilon_{x x}
$$

Using (9) and (10), the strain energy density $\mu$ of the per unit volume can be expressed as

$$
\mu=E \int_{0}^{\varepsilon_{x x}} \bar{\sigma}_{x x} d \varepsilon_{x x}=E \sum_{n=1}^{\infty} \tau^{2 n-2} \int_{0}^{\varepsilon_{x x}} \frac{\partial^{2 n-2} \varepsilon_{x x}}{\partial \bar{x}^{2 n-2}} d \varepsilon_{x x}
$$

In order to facilitate the integration, the strain energy density $\mu$ is divided into three parts and they can be expressed as

$$
\mu=\mu_{1}+\mu_{2}+\mu_{3}
$$

where $\mu_{i}, i=1,2,3$ could be expressed as 


$$
\begin{gathered}
\mu_{1}=\frac{1}{2} E \varepsilon_{\mathrm{xx}}^{2} \\
\mu_{2}=\frac{1}{2} E \sum_{n=1}^{\infty}(-1) \tau^{n+1}\left(\frac{\partial^{n} \varepsilon_{x x}}{\partial \bar{x}^{n}}\right)^{2} \\
\mu_{3}=E \sum_{n=1}^{\infty}\left\{\tau^{2 n+2} \sum_{m=1}^{n}\left[(-1)^{m+1} \frac{\partial^{m} \varepsilon_{x x}}{\partial \bar{x}^{m}} \frac{\partial^{2 n-m+2} \varepsilon_{x x}}{\partial \bar{x}^{2 n-m+2}}\right]\right\}
\end{gathered}
$$

Thus, the total strain energy for the total volume is

$$
U=\int_{V} \mu \mathrm{d} V=\int_{V}\left(\mu_{1}+\mu_{2}+\mu_{3}\right) d V,
$$

where $U$ is the total strain energy, $V$ is the total volume.

The Casimir force for each unit length is [14]

$$
F_{c}=\frac{\pi^{2} \hbar c b}{240(g-w)}
$$

where $\hbar=1.055 \times 10^{-34} \mathrm{Js}$ is the Planck's constant divided by $2 \pi$ and $c=2.998 \times 10^{8} \mathrm{~ms}^{-1}$ is the speed of light. The van der Waals force for each unit length [15] is

$$
F_{v a n}=\frac{A b}{6 \pi(g-\omega)^{3}}
$$

where $A$ is the Hamaker constant.

The electrostatic force for each unit length is

$$
F_{e}=\frac{\varepsilon_{0} b v^{2}}{2(g-\omega)^{2}}\left[1+\frac{0.65(g-\omega)}{b}\right]
$$

where $\varepsilon_{0}=8.854 \times 10^{-12} \mathrm{C}^{2}\left(\mathrm{Nm}^{2}\right)^{-1}$ is the permittivity of vacuum, $v$ is the applied voltage.

Thus the distributed force $p$ can be considered as

$$
p(x)=F_{v a n}+F_{c}+F_{e}
$$

Thus the work done by the distributed force $p$ is

$$
W=\int_{0}^{L} \omega p(x) d x=L^{2} \int_{0}^{1} p(x) \bar{\omega} d \bar{x}
$$

The effective nonlocal elasticity theory is based on the Energy variational method. The nonlocal strain energy and infinite number of the higher-order boundary conditions are derived from the method.

In order to apply the Hamilton's principle, an energy function is introduced

$$
\delta I=\delta U-\delta W=0
$$

By substituting (14) and (19) into (20), we can get $\delta I=\delta U-\delta W$

$$
\begin{gathered}
=\int_{0}^{1}\left\{\frac{E I}{L}\left[-\sum_{n=1}^{\infty}(2 n-3) \tau^{2 n-2} \frac{\partial^{2 n+2} \bar{\omega}}{\partial \bar{x}^{2 n+2}}\right]-L^{2} p\right\} \delta \bar{\omega} d \bar{x} \\
+\frac{E I}{L}\left[\left(\sum_{n=1}^{\infty}(2 n-3) \tau^{2 n-2} \frac{\partial^{2 n+1} \bar{\omega}}{\partial \bar{x}^{2 n+1}}\right) \delta \bar{\omega}\right. \\
\left.-\left(\sum_{n=1}^{\infty}(2 n-3) \tau^{2 n-2} \frac{\partial^{2 n} \bar{\omega}}{\partial \bar{x}^{2 n}}\right) \delta\left(\frac{\partial \bar{\omega}}{\partial \bar{x}}\right)\right]_{0}^{1}
\end{gathered}
$$

$$
\begin{array}{r}
+\frac{E I}{L}\left[\left(\sum_{n=1}^{\infty}(2 n-1) \tau^{2 n} \frac{\partial^{2 n+1} \bar{\omega}}{\partial \bar{x}^{2 n+1}}\right) \delta\left(\frac{\partial^{2} \bar{\omega}}{\partial \bar{x}^{2}}\right)\right. \\
\left.-\sum_{n=1}^{\infty} 2 n \tau^{2 n+2} \frac{\partial^{2 n+2} \bar{\omega}}{\partial \bar{x}^{2 n+2}} \delta\left(\frac{\partial^{3} \bar{\omega}}{\partial \bar{x}^{3}}\right)\right]_{0}^{1} \\
+\frac{E I}{L}\left[\left(\sum_{n=1}^{\infty}(2 n-1) \tau^{2 n+2} \frac{\partial^{2 n+1} \bar{\omega}}{\partial \bar{x}^{2 n+1}}\right) \delta\left(\frac{\partial^{4} \bar{\omega}}{\partial \bar{x}^{4}}\right)\right. \\
\left.-\sum_{n=1}^{\infty} 2 n \tau^{2 n+4} \frac{\partial^{2 n+2} \bar{\omega}}{\partial \bar{x}^{2 n+2}} \delta\left(\frac{\partial^{5} \bar{\omega}}{\partial \bar{x}^{5}}\right)\right]_{0}^{1} \\
+\frac{E I}{L}\left[\left(\sum_{n=1}^{\infty}(2 n-1) \tau^{2 n+1} \frac{\partial^{2 n+1} \bar{\omega}}{\partial \bar{x}^{2 n+1}}\right) \delta\left(\frac{\partial^{6} \bar{\omega}}{\partial \bar{x}^{6}}\right)+\ldots\right]_{0}^{1}
\end{array}
$$

According to the Energy variational method, the variational of the energy function should be zero, and the variational on the boundary is zero, therefore, the governing equations can be written as

$$
-\left[\sum_{n=1}^{\infty}(2 n-3) \tau^{2 n-2} \frac{\partial^{2 n+2} \bar{\omega}}{\partial \bar{x}^{2 n+2}}\right]=\frac{p L^{3}}{E I}
$$

The general solution of (22) is

$\bar{\omega}=C_{1} e^{\bar{x} / \tau}+C_{2} e^{-\bar{x} / \tau}+C_{3} \bar{x}^{3}+C_{4} \bar{x}^{2}+C_{5} \bar{x}+C_{6}-\frac{1}{24} \frac{p L^{3}}{E I} \bar{x}^{4}$

The higher-order boundary conditions consist of natural boundary conditions and geometric boundary conditions can be derived from (21), and each condition for the one situation can be used but not together.

$$
\begin{gathered}
\bar{\omega}(0)=0, \bar{\omega}^{\prime}(0)=0, \bar{\omega}^{\prime \prime}(0)=0, \\
-\bar{\omega}^{\prime \prime}(1)+\tau^{2} \bar{\omega}^{(4)}(1)=0, \\
2 \tau^{4} \bar{\omega}^{(4)}(0)+4 \tau^{6} \bar{\omega}^{(6)}(0)=0, \\
2 \tau^{4} \bar{\omega}^{(4)}(1)+4 \tau^{6} \bar{\omega}^{(6)}(1)=0 .
\end{gathered}
$$

The boundary conditions for fixed-fixed nano-switches are

$$
\begin{gathered}
\bar{\omega}(0)=0, \quad \bar{\omega}(1)=0, \\
\bar{\omega}^{\prime}(0)=0, \quad \bar{\omega}^{\prime}(1)=0, \\
\bar{\omega}^{(4)}(0)=0, \quad \bar{\omega}^{(4)}(1)=0 .
\end{gathered}
$$

\section{NUMERICAL RESUltS AND DiSCUSSION}

\section{A. The Cantilever Nano-Switches}

In the Fig. 3, we have considered the variation of the free standing deflection in different theories under the same conditions. As the value of coordinates $x$ increasing, the deflection decreased as a curve with a single peak. When the nonlocal nanoscale parameter $\tau$ approaches to zero, the curve of the different theories comes closest to coinciding in shape. What's more, at the same point, the deflection of the cantilever under the effective nonlocal elasticity theory is lesser than that under the classical theory. Thus, the cantilever under the effective nonlocal elasticity theory becomes harder than that in classical theory. 


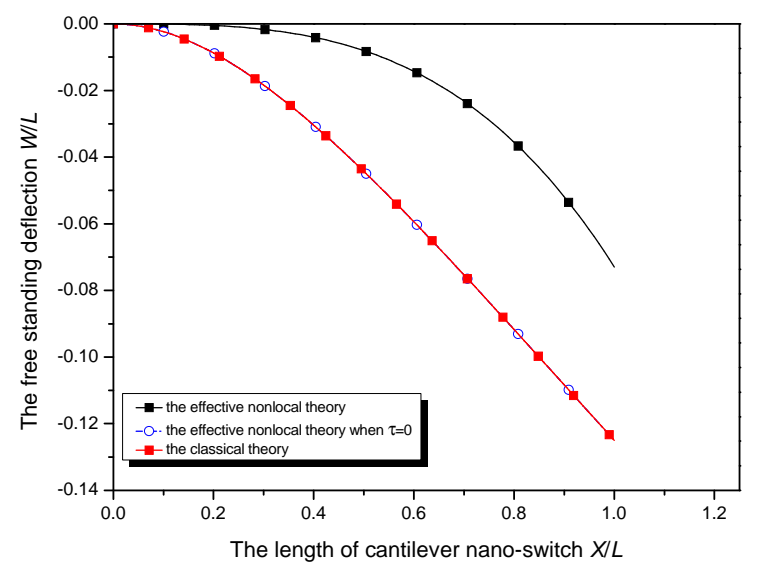

Fig. 3. Variation of the free standing deflection of the cantilever against the length of cantilever nano-switch.

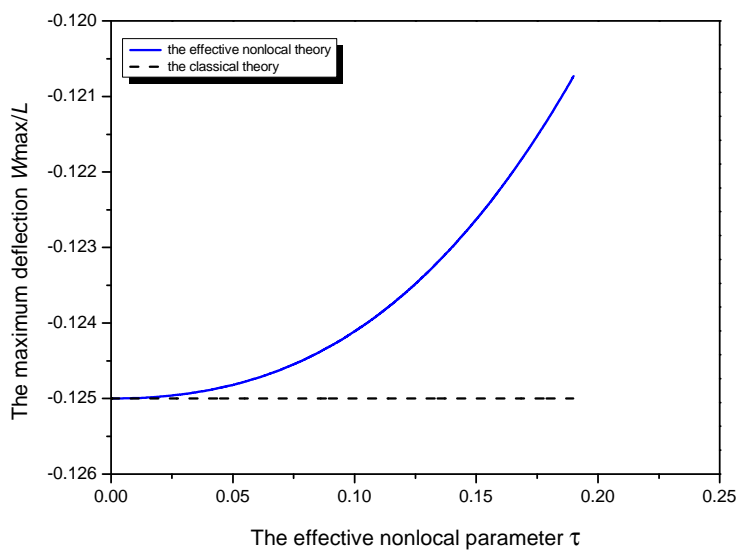

Fig. 4. Variation of the maximum deflection of cantilever against the nonlocal parameter.

In the Fig. 4, we take the effective nonlocal nanoscale parameter $\tau$ into consideration. The effective nonlocal nanoscale parameter $\tau$ is used to show the effective nonlocal effect. In macroscopic states ( $\tau \rightarrow 0$ ), the maximum deflection of cantilever will not respond to changes in the nonlocal parameter. Therefore, the classical theory's curve remains a straight line. What's more, in the nanoscales, the larger the nonlocal parameter is, the smaller the maximum deflection is. In a word, the effective nonlocal elasticity theory makes the cantilever harder.

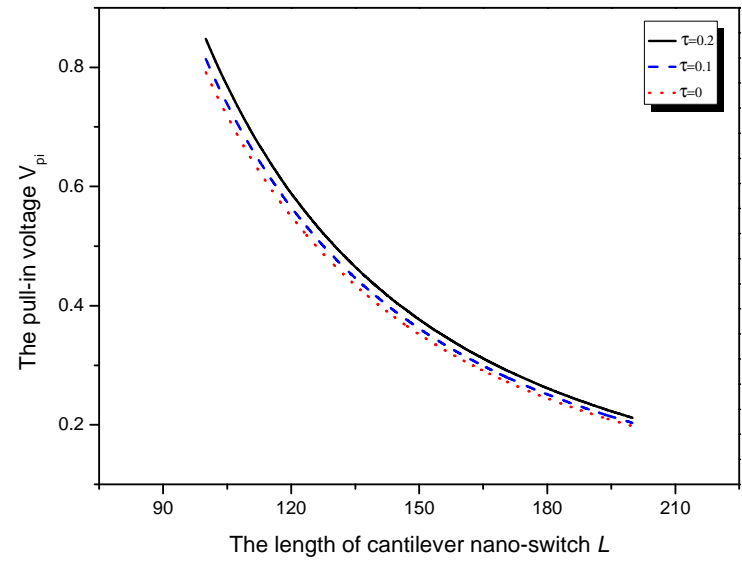

Fig. 5. Variation of pull-in voltage against the length of cantilever nano-switch.

In the Fig. 5, it can be seen that the pull-in voltage increases with the increases of the effective nonlocal parameter $\tau$. It seems that the cantilever has been harder with considering the nanoscale effect. For a given small-scale parameter $\tau$, it may be observed that with increasing the maximum length of the cantilever beam, the pull-in voltage decrease. In addition, in the model, the pull-in voltage behavior depends significantly on the length of cantilever nano-switch.

\section{B. The Fixed-Fixed Nano-Switches}

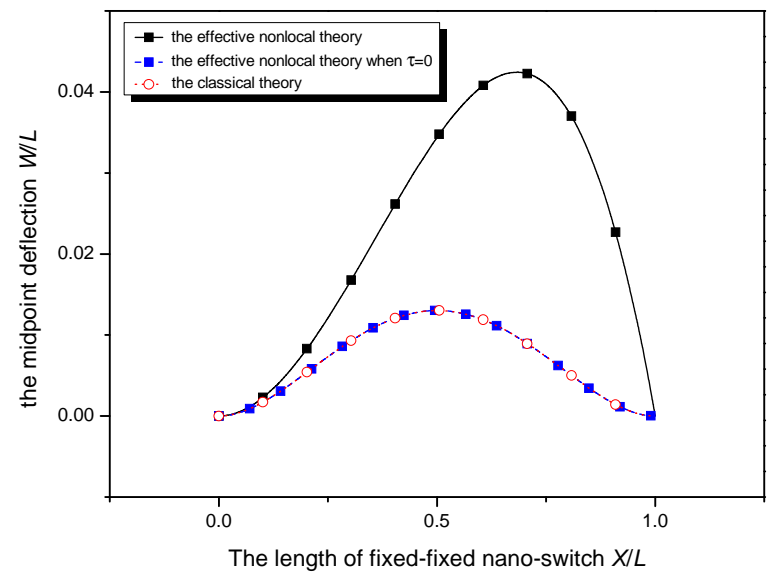

Fig. 6. Variation of the midpoint deflection of the fixed-fixed nano-switch against the length of fixed-fixed nano-switch.

In the Fig. 6, we could figure out that, with considering the effective nonlocal effect, the free standing deflection of the fixed-fixed nano-switch has been changes to even lesser than it without considering the effective nonlocal effect. We conclude that the fixed-fixed nano-switch has become soft in the effective nonlocal theory than it in the classical theory.

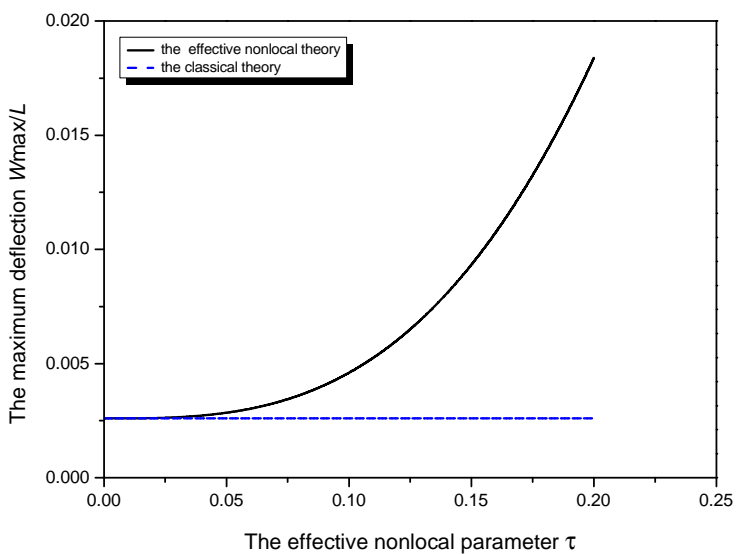

Fig. 7. Variation of the maximum deflection of fixed-fixed nano-switch against the effective nonlocal parameter.

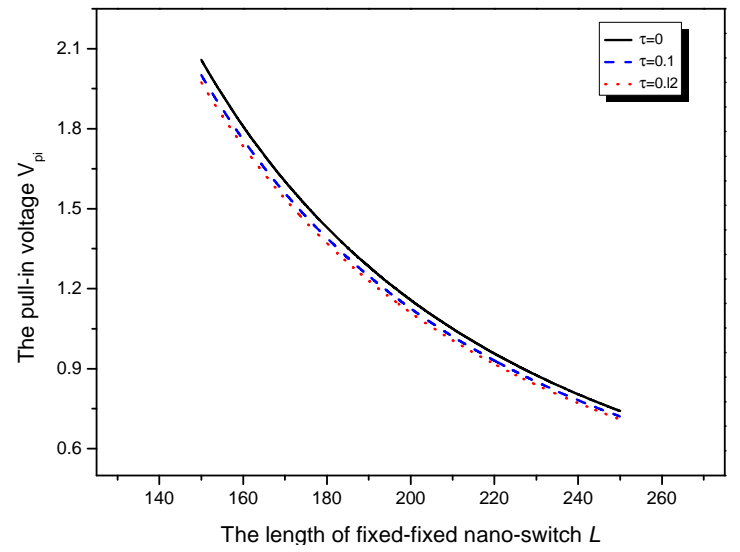

Fig. 8. Variation of pull-in voltage against the length of fixed-fixed nano-switch. 
In the Fig. 7, as the effective nonlocal parameter $\tau$ increases, the maximum deflection of fixed-fixed nano-switch showed different changing tendency varying the theories. In macroscopic states $(\tau \rightarrow 0)$, the maximum deflection of cantilever will not respond to changes in the nonlocal parameter. Therefore, the classical theory's curve remains a straight line. Furthermore, in the nanoscales, the maximum deflection increases while the nonlocal parameter increases. In other words, the fixed-fixed nano-switch has become softer than before.

In the Fig. 8, for a given small-scale parameter $\tau$, we can know that the pull-in voltage decreases as the length of the fixed-fixed nano-switch increases. The length of fixed-fixed nano-switch has a considerable influence on the pull-in voltage. What's more, the effective nonlocal parameter $\tau$ increases during the pull-in voltage decreases. We could infer that the fixed-fixed nano-switch has become softer than before considering the effective nonlocal effect.

\section{CONCLUSIONS}

In this paper, we have studied the effect of small scale on the dynamic behavior of cantilever and fixed-fixed nano-switches using the effective nonlocal elasticity theory. We have introduced the effective nonlocal nanoscale parameter $\tau$ to embody the small scale effect. The governing equations for the dynamic response of the nano-switches including small-scale effect are derived by using the energy variational method.

It is observed that the cantilever and the fixed-fixed nano-switch treated differently under the small scale effect. An increase in the small scale factor leads to higher pull-in voltage for a cantilever nano-switch, results in lower pull-in voltage for a fixed-fixed nano-switch, but does not change the pull-in deflection for both nano-switches. The fixed-fixed nano-switch is more sensitive to the nonlocal effect than the cantilever.

The nonlocal cantilever nano-switch shows stiffening effects due to the small scale effect and tends to become stiffer compared to classical one. Furthermore, the fixed-fixed nano-switch displays softening effects and tends to be softer compared to local one.

\section{REFERENCES}

[1] N. Taghavi and H. Nahvi, "Pull-in instability of cantilever and fixed-fixed nano-switches," European Journal of Mechanics A/Solids, vol. 41, pp. 123-133, September-October 2013.

[2] G. F. Wang and F. Yang, "Postbucking analysis of nanowires with surface effect," Journal of Applied Letters, vol. 109, pp. 063535 063535-4, April 2011.

[3] Z. Y. Ou and S. D. Pang, "Fundamental solutions to hertzian contact problems at nanoscale," Acta Mechanics, vol. 224, pp. 109-121, January 2013.

[4] T. Murmu and S. C. Pradhan, "Small-scale effect on the free in-plane vibration of nanoplates by nonlocal continuum model," Physica E, vol. 41, pp. 1628-1633, May 2009.
[5] A. C. Eringen, "On differential-equations of nonlocal elasticity and solutions of screw dislocation and surface-waves," Journal of Applied Physics, vol. 54, pp. 4703-4710, 1983.

[6] U. B. C. O. Ejike, "The plane circular crack problem in the linearized couple-stress theory," International Journal of Engineering Science, vol. 7, pp. 947-961, September 1969.

[7] Y. T. Beni, M. R. Abadyan, and A. Noghrehabadi, "Investigation of size effect on the pull-in instability of beamtype NEMS under van der waals attraction," Procedia Engineering, vol. 10, pp. 1718-1723, June 2011.

[8] J. Yang, X. L. Jia, and S Kitipornchai, "Pull-in Instability of nano-switches using nonlocal elasticity theory," Journal of Physics D: Applied Physics, vol. 41, no. 3, pp. 035103, January 2008.

[9] J. S. Peng, G. B. Luo, L. Yang, and J. Yang, "Pull-in instability behaviour of nanoscale actuators using nonlocal elasticity theory," Advanced Materials Research, vol. 468-471, pp. 2755-2758, February, 2012.

[10] B. L. Wang, S. J. Zhou, J. F. Zhao, and X. Chen, "A size-dependent kirchhoff micro-plate model based on strain gradient elasticity theory," European Journal of Mechanics A/Solids, vol. 30, pp. 517-524, July-August 2011.

[11] T. Mousavi, S. Bornassi, and H. Haddadpour, "The effect of small scale on the pull-in instability of nano-switches using DQM," International Journal of Solids and Structures, vol. 50, pp. 1193-1202, May 2013.

[12] K. F. Wang and B. L. Wang, "Influence of surface energy on the non-linear pull-in instability of nano-switches," International Journal of Non-Linear Mechanics, vol. 59, pp. 69-75, January 2014.

[13] A. C. Eringen, Nonlocal Continuum Field Theories, 1st ed. New York: Springer-Verlag Inc., 2002, ch. 6, pp. 71-90.

[14] L. K. Steven, "The casimir force: background, experiments, and applications," Reports on Progress in Physics, vol. 68, pp. 201-236, January 2005.

[15] I. N. Jacob, Intermolecular and Surface Forces, 3rd ed. Amsterdam: Elsevier Inc., 2011, ch. 6, pp. 107-132.

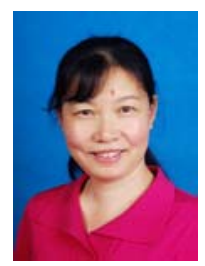

Bo Chen was born in February 1965. She has been working as a senior engineer in the School of Mechanical and Electronical Engineering, Lanzhou University of Technology, Lanzhou, China. Her mainly research interest is mechanical design theory and mechatronics.

She has studied the drilling machine and had published an article about the study of the control system of automatic drilling.

She has studied the stability analysis of the drilling machine and had make certain achievements.

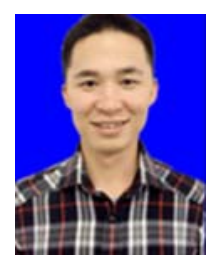

Liang Tan was born in January 1987. He has studied as a master in the School of Mechanical and Electronical Engineering, Lanzhou University of Technology, Lanzhou, China. His mainly research interest is Nano-mechanical.

He has studied the nanostructures and the dynamics of the nano-beams.

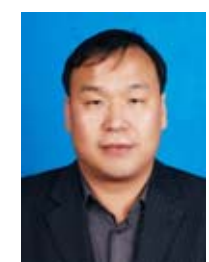

Zhiying Ou was born in July 1970. He has been working as a professor in the School of Science, Lanzhou University of Technology, Lanzhou, China. His mainly research interest is Mathematical methods in solid mechanics.

He had studied the surface mechanical behavior of materials and structures of micro and nano-scale and had published several articles about the study of the mechanical behavior of the NEMS. 